\title{
Twenty Years of Galactic Observations in Searching for Bursts of Collapse Neutrinos with the Baksan Underground Scintillation Telescope
}

\author{
E.N.Alexeyev*, L.N.Alexeyeva \\ Institute for Nuclear Research, Russian Academy of Sciences, \\ pr.Shestidesyatiletiya Oktyabrya 7a, Moscow, 117312 Russia \\ *e-mail: alexeyev@ms1.inr.ac.ru \\ Received: January 29, 2002
}

\begin{abstract}
The results of twenty-year-long Galactic observations in neutrino radiation are summarized. Except for the recording of a neutrino signal from the supernova SN 1987A, no Galactic bursts of collapse neutrinos have been detected. An upper bound on the mean frequency of gravitational collapses in our Galaxy was obtained, $f_{\text {collapse }}(90 \%$ confidence $)<0.13 \mathrm{yr}^{-1}$.
\end{abstract}

PACS numbers: 95.30.Cq, 95.55.Vj, 95.85.Ry, 97.60.Bw, 97.60.Ld

\section{Introduction}

In 1933, Wolfgang Pauli introduced a new neutral particle of low or zero mass to save the law of conservation of energy in nuclear beta decays [1]. This particle was experimentally observed only twentyfive years later by Reines and Cowan [2]. The interactions of neutrinos and their role in particle physics, astrophysics, and cosmology have been studied with an increasing intensity ever since. The discovered particle turned out to be so amazing that it allowed one not only to study nuclear processes on Earth and in its atmosphere but also to look into stellar objects, because it is highly penetrating due to the weakness of its interactions with matter.

Progress in theoretical and experimental research has led to the development of many detectors designed to search for and record terrestrial and extraterrestrial neutrinos. Since the energy sources in stars are nuclear reactions, they also emit neutrinos. A new line of research emerged - neutrino astronomy. However, a constant flux of low-energy neutrinos is very difficult to detect, as evidenced by long-term experiments to study neutrino fluxes from the Sun, the star closest to the Earth [3, 4, 5].

Back in 1934, Baade and Zwicky [6] suggested the existence of neutron stars and came up with the idea that these are formed during supernova explosions. Thirty years later, in 1965, Zel'dovich and Gusseinov [7] concluded that a short burst of high-energy neutrinos could arise when matter is neutronized in the gravitational core collapse of a massive star. In the same year, Domogatsky and
Zatsepin [8] pointed out an experimental possibility of searching for such neutrino bursts. In 1966, Colgate and White [9] surmised that neutrinos could play a crucial role in supernova explosions. Almost concurrently, the discovery of pulsars in the Crab Nebula [10, which is the supernova remnant observed by Chinese astronomers in 1054, and in Vela 11] late in the fall of 1968 provided evidence for the formation of neutron stars during supernova explosions. Subsequent observations and data analysis confirmed this conclusion and gave an insight into the physical processes that underlie these phenomena 12, 13].

In the succeeding years, both a theory for the final stages of stellar evolution and experiments to search for bursts of such collapse neutrinos were intensively developed. The explosion of the supernova SN 1987A on February 23, 1987, was a special milestone in the development of the two lines of research.

The first experiments to search for neutrino bursts from gravitational stellar core collapses were begun in 1973 by Pennsylvania-Texas-Turin collaboration. These were three small water Cherenkov facilities with target masses of about $20 \mathrm{t}$ each and with particle detection energy thresholds of 20 $\mathrm{MeV}$ : the first was at the Homestake Mine at a depth of 4400 meters of water equivalent (m.w.e.) 14], the second was at a mine in Ohayo at a depth of 1800 m.w.e. [15], and the third was in the road tunnel under Mont Blanc at a depth of 4270 m.w.e. [16]. They did not work long, only a few years. Al- 
Journal of Experimental and Theoretical Physics, Vol. 95, No.1, 2002, pp. 5-10

though no expected burst of collapse neutrinos was detected, they made the first step on the long way to 1987.

The Baksan underground scintillation telescope (Northern Caucasus, Russia) [17], the LSD scintillation facility under Mont Blanc (Italy) [18], the scintillation detector at the salt mine in the town of Artemovsk (Ukraine) [18], and the water Cherenkov detectors Kamiokande II (Japan) [19] and IMB (USA) [20] belong to the second generation of collapse-neutrino detectors with target masses of several hundred tons, which began their operation in 1977-1980. The last two detectors were specially designed to search for proton decays, but they proved to be incapable of recording collapse neutrinos.

Before 1987, the theoretical models for the late evolutionary stages of massive stars were onedimensional calculations of spherically symmetric nonmagnetic nonrotating configurations. The basic neutrino-radiation parameters expected during the gravitational core collapse of a massive star and the cooling of a newborn neutron star were formulated: the total neutrino-radiation energy is $(2 \div 5) 10^{53} \mathrm{erg}$, , the mean electron-neutrino energy is $(8 \div 12) \mathrm{MeV}$, the fraction of electron antineutrinos in the total radiation is $(0.16 \div 0.25)$ and the burst duration is $(10 \div 20) \mathrm{s}$ [21, 22, 23, 24].

The recording of a neutrino signal from a typeII supernova exploded in the Large Magellanic Cloud, a neighboring galaxy, by three facilities, Kamiokande II [25], IMB [26], and the Baksan telescope [27], on February 23, 1987, first confirmed the basic ideas of the general theoretical pattern of gravitational collapse with the formation of a neutron star. At the same time, however, it raised many questions both for theory and for experiment.

Observations of the expanding envelope of the supernova remnant show that the explosion was asymmetric, with an unusual shape of the remnant, with matter mixing [28, 29], with an as yet undiscovered pulsar [30] or with a candidate for a pulsar with unusual properties 31, 32]. All of this led to rapid progress in the theoretical modeling of processes inside stars and in understanding the many processes in core collapse and in the stellar envelope, as well as to a complication of the collapse pattern [33, 34, 35], particularly when rotation and strong magnetic fields are included in the calculations [36]. However, not all of the questions have been answered as yet. In particular, the stellar-envelope ejection mechanism and, what is important for experimenters, the complete temporal structure of the neutrino luminosity are still problems to be solved. In addition, the general-relativity [37] and weakmagnetism [38] effects, which have not yet been included in the calculations and which may lead to a higher neutrino luminosity, as well as a possible nonzero neutrino mass, which would cause the neutrino spectra to change [39], are being discussed.

The development of methods for analyzing experimental data with small statistics and using them to study the neutrino signals recorded on February 23,1987 , by three facilities also led authors to conclude that the neutrino radiation has a more complex temporal structure 40].

An important lesson drawn from the history of experimental observation of the neutrino signal from SN 1987A is that the facilities capable of recording collapse neutrinos improved and adjusted their physical parameters and are combined into a single network, SNEWS (SuperNova Early Warning System), to form a coincidence trigger for their signals (when they appear) that will notify observatories of other types of radiation of the beginning of supernova observations 41].

In addition, it became clear that detectors with an even larger target mass sensitive to all types of neutrino are required to reliably and completely record the expected Galactic neutrino burst. This is how the third-generation neutrino detectors with a characteristic target mass of about $1 \mathrm{kt}$ or higher and with low event detection energy thresholds (about $5 \mathrm{MeV}$ ) emerged. When a Galactic burst of collapse neutrinos occurs, the most powerful of these detectors will record over 8500 neutrinos of all types (SuperKamiokande, the target mass is $32 \mathrm{kt}$ of water) 42, about 1000 neutrinos of all types in the SNO detector with heavy and light water (the total target mass is $2.4 \mathrm{kt}$ ) 43, and over 350 neutrinos of all types in the LVD scintillation detector 44]. Apart from the long-operating LSD [18 and MACRO 45] scintillation facilities, which will also record about 100 or more Galactic neutrinos each, the Amanda facility in the ice at the South Pole in Antarctica [46] was included in the network of collapse- neutrino observations in 1997. A number of new detectors are also prepared to be launched or are being designed 47 .

Thus, of particular importance is the so unexpected phenomenon of a type-II or, possibly, type$\mathrm{Ib} / \mathrm{c}$ supernova [48] in our Galaxy. At least seven neutrino detectors, one of which is the Baksan underground scintillation telescope, were prepared for its recording.

\section{The Baksan Underground Scintillation Telescope}

The Baksan underground scintillation telescope is an instrument for a broad range of scientific research, with one of its experiments being the search for neutrino burst from supernova explosions. The 


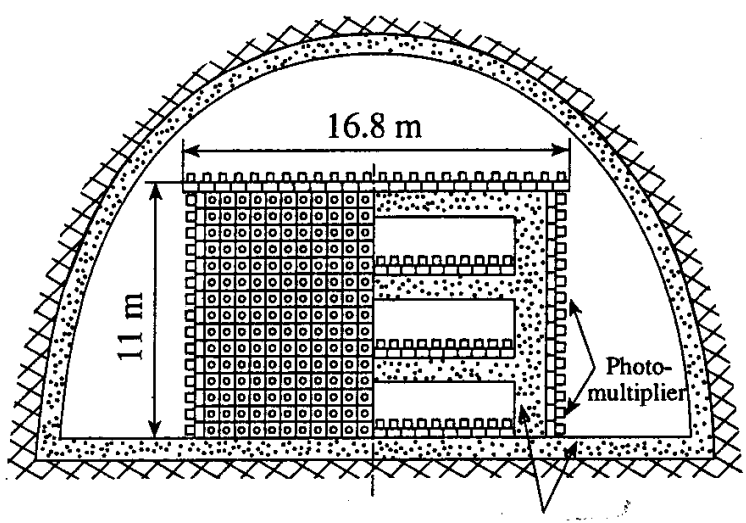

Low-radioactivity concrete

Figure 1: A schematic view of the Baksan underground scintillation telescope.

telescope is located in the Northern Caucasus, at the foot of the Mount Elbrus, in the tunnel under the Andyrchi Mountain at a depth of 850 m.w.e.. A general view of the telescope is schematically shown in Fig.1, and the facility is described in detail in 49 . Here, we briefly recall its basic parameters.

The telescope consists of 3150 standard detectors. These detectors form a closed configuration with two internal layers that comprise four vertical planes and four horizontal planes. Each standard detector is $70 \times 70 \times 30^{3}$ in size, is filled with an organic $C_{n} H_{2 n+2},(n \sim 9)$ scintillator, and is viewed by one photomultiplier with a photocathode diameter of $15 \mathrm{~cm}$. The total target mass is $330 \mathrm{t}$, and the target mass enclosed in the three internal (starting from the lower horizontal plane) layers is $130 \mathrm{t}$ (1200 standard detectors). The charged-particle detection energy thresholds are 8 and $10 \mathrm{MeV}$ for the horizontal and vertical planes, respectively. The energy reference for standard-detector measurements is the amplitude of the most probable energy release when cosmic-ray muons pass through it $(50 \mathrm{MeV})$. Accordingly, the detector thresholds are given in fractions of the amplitude of this energy release.

An intense burst of neutrinos of all types is expected during a type-II supernova explosion. However, the vast majority of the events recorded with the Baksan telescope will be produced in inverse beta-decay reactions,

$$
\overline{\nu_{e}}+p \rightarrow n+e^{+} .
$$

The contribution from the remaining types of neutrinos to the total recorded signal will be minor 49.

Since the telescope is located at a relatively small depth, a special event-selection method is used to significantly reduce the background from the cosmic-ray muons that cross the facility in our experiment. This methods is based on an enor- mous energy difference between the muons and the positrons formed from collapse electron antineutrinos. Passing through the telescope, the muons leave a track from several triggered detectors, while the positrons formed in a particular detector will lose all of their energy almost entirely in this detector. Thus, the main event-selection criterion in our program, - one and only one detector from 3150, - implies the selection of single standard-detector triggerings; i.e., at the program marker, data on the state of all devices in the facility at the triggering time of one and only one detector in it is written to the online computer memory and then to storage.

As a result, the neutrino signal from a supernova explosion on the facility will appear as a series of events from singly triggered detectors during the neutrino burst.

The count rate from single background events, the statistical clumps of which can imitate the expected signal, was not constant with time at the beginning of the experiment on the telescope, because operations were continuously performed to remove one or another background source, which caused a continuous reduction in the total count rate of single events. Figure 2 shows time variations in the yearly mean count rate of single detector triggerings summed over three internal planes (the lower and the next two planes in Fig.1) in which $130 \mathrm{t}$ of scintillator are contained in 1200 detectors. The data from this part of the facility, i.e., the total number of single triggerings within a given time interval, act as a trigger in the offline analysis of information from the telescope: when an unusual signal was detected in this part of the telescope (i.e., when the number of events was larger than a given number), information from the entire facility was analyzed offline.

Figure 2 also indicates the time intervals when the particle detection energy threshold on the telescope was reduced. As we see from this figure, the background after 1985, when the main operations to remove the strongest noise were finished, was virtually constant within a certain detection energy threshold. Its subsequent modest reduction resulted from the continuous operations to improve the physical parameters of the facility.

The time when the explosion of SN 1987A occurred is shown in the same figure.

If the core of a massive star collapses at the Galactic center to form a neutron star, then one might expect over 50 events on the telescope from the interactions of electron antineutrinos in the 130-t scintillator or over 100 events in the entire telescope. The current information is analyzed by the method of a $20-\mathrm{s}-$ long time interval sliding from event to event with the total threshold number of events in the signal equal to 5. For detection of a signal with a given or larger number of events, 


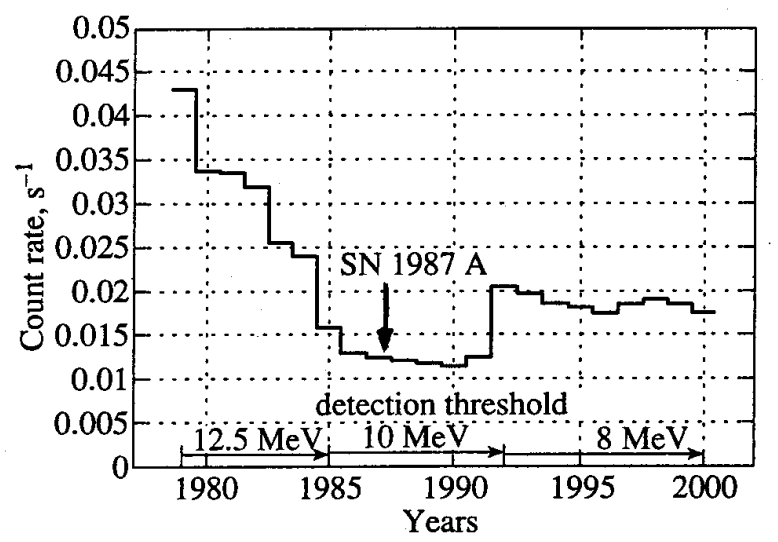

Figure 2: Time variations in the yearly mean count rate of single triggerings of 1200 detectors in the three telescope internal planes (the target mass is 130t). The particle detection energy thresholds in different years are shown. The arrow indicates the explosion of SN 1987A on February 23, 1987.

40 rather than $20 \mathrm{~s}$ of information with complete data on each event (coordinates on the telescope, time, energy amplitude, the number of detector triggerings in the signal over the current day, and photomultiplier-pulse duration) is analyzed. A signal would be considered to be a serious candidate for collapse detection if nine or more events were observed for $20 \mathrm{~s}$ in the telescope target mass of $130 \mathrm{t}$ (internal layers). No such signal was detected over the entire period of observations with the telescope.

The telescope was constructed late in 1977. However, the physical experiments began later, because it took time for us to figure out how the instrument behaved. The facility has been operating almost continuously under the program of search for collapse neutrinos (the program was called Collapse) since the mid-1980. One maintenance day per week, when the necessary repair and maintenance operations are performed on the facility, and the emergency situations of seasonal power cuts or online computer replacement constitute an exception. So, the total time of Galactic observation accounts for $90 \%$ of the calender time.

Thus, information from the telescope was accumulated under the program of search for collapse neutrinos, but there was no expected neutrino signal. The history of detecting the neutrino signal from SN 1987A in the Baksan data and the related dramatic searches for the cause of the then unexpected clock error have long been published [28, 49. A new clock with a self-contained power supply has been in operation since February 1988, providing a $1-$ ms accuracy of determining the absolute time.

\section{Results of the Observations}

When the Baksan experiment to search for collapse neutrinos began, the frequency of expected signals was of great importance. Observations of historical supernovae in our Galaxy, supernovae in other galaxies, stellar statistics, pulsar statistics, and even the thermoluminescence of samples of bottom sediments were used in the literature to determine the time interval between events in which supernovae, neutron stars, or black holes were expected to be formed.

At that time, the most optimistic estimate of this interval was obtained from an analysis based on observations of 149 pulsars, which predicted the birth of one pulsar approximately every six years [50]. Although a later reanalysis of the pulsar birth rate yielded a different estimate of this interval, about 30 years [51], we still hoped for a positive result at the beginning of our experiment, especially since the last supernova was observed on Earth 400 years ago.

Another optimistic result was obtained by analyzing stellar statistics: when calculating the total star death rate with the ultimate formation of pulsars, black holes, supernovae, and any other possibilities, except white dwarf, the interval between such events was found to be $9_{-3}^{+2}$ years [52]. The estimates for the rate of type-II supernovae in our Galaxy by other authors give a large spread in the predictions of its mean value. This appears to be attributable to a large number of assumptions used and approximations, for example, about the luminosity of the Galaxy [53], its morphological type [54], the initial mass function [55], the fraction of detected pulsars [56], and many others [57, 58, 59, 60].

Subsequently, the relationships between supernova statistics and galactic evolution were analyzed and it was concluded that the supernova rates were functions of cosmic time [61, 62]. In addition, a study of the light curves for SNe Ib/c showed them to be similar to the light curves for type-II supernovae [48], led one to conclude that the $\mathrm{SN} \mathrm{Ib/c}$ progenitor was a massive star, and suggested that neutron stars could also be formed during their explosions.

Recent estimates of the supernova rate from stellar statistics show that the following number of such events may be expected in our Galaxy [63, 64]: $2_{-1}^{+1}$ for $\mathrm{SNe} \mathrm{Ib} / \mathrm{c}$ and $12_{-6}^{+6}$ for SNe II in 1000 years. Recently, however, the same authors improved these Galactic values [65]: $1.5_{-1.0}^{+1.0}$ for $\mathrm{SNe} \mathrm{II}+\mathrm{Ib} / \mathrm{c}$ in 100 years or about one supernova with the formation of a neutron star every 50 years. The latter value is almost equal to this interval previously estimated to be $10-50$ years 66 . when the above possible sources of discrepancies are taken into account. 
In general, it is clear that the sought-for rate estimated from stellar statistics lies within the range $10^{-2}-3 \cdot 10^{-2}$ per year or with a mean estimate of the interval between supernovae equal to $47_{-12}^{+12}$ years 67.

In recent years, it has been found from the statistics of pulsars that they are formed, on average, once every 60-330 years [68], although it is still unclear in what systems the pulsars of a particular class are born 69, 70.

Thus, recent estimates for the rates of supernova explosions and pulsar formation lead us to conclude that this event is rare and that the spread in estimates is large. Therefore, it becomes all the more necessary to obtain the result from direct observations of these events with neutrino detectors.

The Baksan telescope has been observing the Galaxy since June 30, 1980. Because of all the operations performed to increase its sensitivity to the expected burst of collapse neutrinos, the telescope views the entire Galaxy [49. The calendar time of the Collapse experiment is 19.75 years, while the total live observing time is 17.6 years. No signal, except SN 1987A in the Large Magellanic Cloud, that could be reliably interpreted as a burst of Galactic electron antineutrinos was detected with the facility over this period.

An upper bound on the mean frequency of gravitational collapses in the Galaxy can be obtained from the observing time. If we denote the mean frequency of collapses by $f_{\text {collapse }}$ and if we assume that, first, their frequency (as rare events) obeys the Poisson law and, second, the probability of missing the signal is less than $10 \%$ at $90 \%$ confidence, then we derive the following inequality for the total live observing time $\mathrm{T}=17.6$ years:

$$
e^{-f_{\text {collapse }} T}<0.1 \text {, }
$$

whose solution forf $f_{\text {collapse }}$ yields a bound on the frequency of collapses in the Galaxy,

$$
f_{\text {collapse }}(90 \% \text { confidence })<0.13 \mathrm{yr}^{-1} \text {. }
$$

Hence, the mean time interval $\Delta T_{\text {collapse }}$ between the expected Galactic events exceeds

$$
\Delta T_{\text {collapse }}(90 \% \text { confidence })>7.7 \text { years. }
$$

Thus, the first twenty years on the path to detecting the first neutrino burst in our Galaxy have been traversed, although this may prove to be only a small part of the required path.

\section{Acknowledgments}

We wish to thank the staff of the Baksan neutrino observatory, those who are still working and those who have left it, for a long and fruitful collaboration in this experiment. This study was supported by the Russian Foundation for Basic Research (project no. 00-02-17778).

\section{References}

[1] W.Pauli, Noyaux Atomiques (VI Conseil de Physique Solvay, 1933), Paris, p.324 (1934).

[2] F.Reines, C.L.Cowan, Phys.Rev. 90, 492 (1953); 113, 273 (1959).

[3] K.Lande, B.Cleveland, T.Daily et al., AIP Conf. Proc. 243, 1122 (1992).

[4] V.N.Gavrin, Nucl. Phys. Proc. Suppl. 91, 36 (2001).

[5] W.Hampel, Phys.Lett. B447, 127 (1999).

[6] V.Baade, F.Zwicky, Phys. Rev. 45, 138 (1934).

[7] Ya.B.Zel'dovich and O.Kh.Gusseinov, Dokl. Akad. Nauk SSSR 162, 791 (1965) [Sov.Phys.Dokl. 10, $524(1965)]$.

[8] G.V.Domogatsky, G.T.Zatsepin, Proc 9th ICRC, London, UK, 2, 1030 (1965).

[9] S.A.Colgate, R.H.White, Astrophys.J. 143, 626 (1966).

[10] W.J.Cocke, H.J.Disney, D.J.Taylor, Nature, 221, 525 (1969).

[11] M.I.Large, A.E.Vaugham, B.Y.Mills, Nature, 220, 340 (1968).

[12] G.G.Pavlov, D.Sanwal, G.P.Garmire, V.E.Zavlin, ASP Conference Series (2002), "The Puzzling Compact Objects in Supernova Remnants" astro$\mathrm{ph} / 0112322$

[13] V.M.Kaspi, D.J.Helfand, ASP Conference Series (2002), "Constraining the Birth Events of Neutron Stars" astro-ph/0201183.

[14] K.Lande, G.Bozoki, W.Frati et al., Nature, 251, 485 (1974)

[15] M.Deabyne, W.Frati, K.Lande et al., Proc 16th $I C R C$, Kyoto, Japan, 13, 325 (1979).

[16] G.Bozoki, Proc 13th ICRC, Denver, USA, 3, 1994 (1973).

[17] E.N.Alexeyev, L.N.Alexeyeva, A.E.Chudakov, Proc. 16th ICRC, Kyoto, Japan, 10, 282 (1979).

[18] A.E.Chudakov, O.G.Ryazhskaya, Proc. Int. Conf. "Neutrino 77", Baksan Valley, USSR, Nauka, Moscow, 1, 155 (1978).

[19] K.Arisaka, K.Hirata, T.Kajita et al., Phys. Soc. Japan 54, 3213 (1985). 
[20] R.M.Bionta, G.Blewitt, G.B.Bratton et al., Phys. Rev. Lett. 51, 27 (1983).

[21] V.S.Imshennik and D.K.Nadezhin, Itogi Nauki Tekh., Ser.: Astron. 21, 63 (1982).

[22] B.Bowers, J.R.Wilson, Astrophys.J., 263, 366 (1982).

[23] E.E.Salpeter, S.L.Shapiro,Astrophys.J., 251, 311 (1981).

[24] A.Burrows, T.L.Mazurek, Nature, 301, 315 (1983).

[25] K.S.Hirata, T.Kajita, M.Koshiba et al., Phys. Rev. Lett. 58, 1490 (1987).

[26] R.M.Bonita, G.Blewitt, C.B.Bratton et al., Phys. Rev. Lett. 58, 1494 (1987).

[27] E.N.Alekseev, L.N.Alekseeva, V.L.Volchenko, et al., Pis'ma Zh.Eksp.Teor.Fiz. 45, 461 (1987) [JETP Lett. 45, 589 (1987)]; E.N.Alexeyev, L.N.Alexeyeva, I.V.Krivosheina et al., Phys. Lett. B205, 209 (1988).

[28] N.Panagia, "Supernovae", Invited Lecture at the International Summer School "Experimental Physics of Gravitational Waves", Urbino (Italy), 6-19 September, 1999, eds. G. Calamai, M. Mazzoni, R. Stanga \& F. Vetrano,, World Scientific Singapore, astro-ph/0003083.

[29] A.Fassia, W.P.S.Meikle, J.Spiromilio, Mon. Not. R. Astron. Soc. in press (2002), "Spectroscopy of SN 1987A at 0.9-2.4 $\mu \mathrm{m}$ : Days 1348-3158", astro$\mathrm{ph} / 0112516$.

[30] S.Park, D.N.Burrows, G.P.Garmire et al., Astrophys.J. in press (2002), "Monitoring the Evolution of the X-ray Remnant of SN 1987A", astro-ph/ 0111116 .

[31] J.Middleditch, J.A.Kristian, W.E.Kunkel et al., New Astronomy 5, 243 (2000).

[32] S.Nagataki, K.Sato, Prog. Theor. Phys., 105, 429 (2001).

[33] H.-Thomas Janka, Astron. Astrophys., 368, 527 (2001).

[34] H.-Thomas Janka, K.Kifonidis, M.Rampp, Lect. Notes Phys. 578, 333 (2001).

[35] V.M.Suslin, S.D. Ustyugov, V.M.Chechetkin, and G.P.Churkina, Astron. Zh. 78, 281 (2001) [Astron. Rep. 45, 241 (2001)].

[36] J.C.Wheeler, D.L.Meier, J.R.Wlson, "Asymmetric Supernovae from Magneto-Centrifugal Jets", astro-ph/0112020.

[37] S.W.Bruenn, K.R.DeNisco, A.Mezzacappa, "General Relativistic Effects in the Core Collapse Supernova Mechanism", astro-ph/0101400.
[38] C.J.Horowitz, "Weak magnetism for anti-neutrinos in supernovae", astro-ph/0109209.

[39] K.Takahashi, M.Watanabe, K.Sato, T.Totani, Phys. Rev. D64, 093004 (2001).

[40] Th.J.Loredo, Don Q.Lamb, "Bayesian analysis of neutrinos observed from supernova SN-1987A", astro-ph/0107260.

[41] Alec Habig, *College Park 1999, Cosmic explosions*, 169 (1999).

[42] J.F.Beacom, P.Vogel, Phys. Rev. D58, 053010 (1998).

[43] C.J.Virtue, Nucl. Phys. Proc. Suppl. 100, 326 (2001).

[44] M.Aglietta, Nuovo Cim. A105, 1793 (1992).

[45] S.Ahlen, Astropart. Phys. 1, 11 (1992).

[46] J.Ahrens, X.Bai, G.Barouch et al., Astropart. Phys. 16, 345 (2002).

[47] K.Scholberg, Nucl. Phys. Proc. Suppl. 91, 331 (2000).

[48] A.V.Filippenko, "Optical Observations of Type II Supernovae", to appear in "Cosmic Explosions" eds. S. Holt and W. W. Zhang (New York: American Institute of Physics), 2000, astro-ph/0002264.

[49] E.N.Alekseev, L.N.Alekseeva, V.I.Volchenko, et al., Zh. Eksp. Teor. Fiz. 104, 289 (1993) [JETP 77, 339 (1993)].

[50] J.H.Taylor, R.N.Manchester, Astrophys. J. 215, 885 (1977).

[51] V.Trimble, Rev. Mod. Phys. 54, 1183 (1982).

[52] J.N.Bahcall, T.Piran, Astrophys. J. Lett. 267, L77 (1983).

[53] S.van den Bergh, R.D.McClure, R.Evans, Astrophys. J. 323, 44 (1987).

[54] E.Cappellaro, M.Turatto, Astron. Astrophys. 190, 10 (1988).

[55] O.G.Richter, M.Rosa, Astron. Astrophys. 206, 219 (1988).

[56] R.Narayan, K.J.Schaudt, Astrophys. J. Lett. 325, L43 (1988).

[57] S.van den Bergh, Astron. Astrophys. 231, L2 (1990).

[58] D.Battacharya, J. Astrophys. Astr. 11, 125 (1990).

[59] B.N.G.Guthrie, Astron. Astrophys. 234, 84 (1990).

[60] G.A.Tammann, A.Schroeder, Astron. Astrophys. 236, 149 (1990). 
[61] P.Madau, "Galaxy Evolution and the Cosmic Rate of Supernovae", to appear in "The Young Universe: Galaxy Formation and Evolution at Intermediate and High Redshift", (1998), S. D'Odorico, A. Fontana \& E. Giallongo (editors), PASP, astro$\mathrm{ph} / 9801005$.

[62] P.Madau, M.D.Valle, N.Panagia, "On the evolution of the cosmic supernova rates", submitted to Mon. Not. Roy. Astron. Soc., astro-ph/9803284.

[63] E.Cappellaro, M.Turatto, D.Yu.Tsvetkov et al., Astron. Astrophys. 322, 431 (1997).

[64] E.Cappellaro, R.Evans, M.Turatto, Astron. Astrophys. 351, 459 (1999).

[65] E.Cappellaro, "Supernova types and rates", invited review at the meeting: "The influence of binaries on stellar population studies", ed. D. Vanbeveren (Brussels 21-25 Aug. 2000), astro$\mathrm{ph} / 0012455$.

[66] S.van den Bergh, G.A.Tammann, Annual Reviews of Astronomy and Astrophysics, 29, 363 (1991).

[67] G.A.Tammann, W.Loeffler, A.Schroeder, Astrophys. J. Suppl. 92, 487 (1994).

[68] A.G.Lyne, Mon. Not. R. Astron. Soc., bf 295, 743 (1998).

[69] S.F.Portgies Zwart, E.P.J.van den Heuvel, New Astron. 4, 355 (1999).

[70] D.R.Lorimer, "Neutron Star Birth Rates", to appear in the proceedings of the NATO ASI "The Neutron Star - Black Hole Connection", astroph/9911519. 